\title{
High- $Q$ Optical Cavities at Visible Wavelengths in Photonic Crystals in the Anderson-localized regime
}

\author{
Tom Crane*, Oliver J. Trojak*, Luca Sapienza \\ Department of Physics and Astronomy, University of Southampton, SO17 1BJ, Southampton, United Kingdom \\ * equal contributors \\ Author e-mail address: l.sapienza@soton.ac.uk
}

\begin{abstract}
We demonstrate Anderson localization of visible light in silicon nitride photonic crystal waveguides. We measure photoluminescence resonances due to disorder-induced light localization showing quality factors of $\approx 10000$ that exceed engineered $2 \mathrm{D}$ photonic crystal cavities.

OCIS codes: (350.4238) Nanophotonics and photonic crystals; (290.4210) Multiple scattering; (300.6280) Spectroscopy, fluorescence and luminescence
\end{abstract}

\section{Introduction}

Confining light in optical cavities is of tremendous interest for a variety of applications, spanning from the investigation of fundamental light-matter interactions to applications in sensing, imaging and quantum information technology [1]. The development of efficient optical cavities operating at visible wavelengths is particularly important for the study of cavity quantum electrodynamics effects with defect centers in diamond and to control and boost the emission dynamics of organic molecules and colloidal quantum dots.

Engineered two-dimensional photonic crystal cavities in silicon nitride, however, have shown quality factors limited to values of the order of 1000 [2,3]. Such low confinement efficiencies, compared to longer wavelength devices, can mostly be attributed to losses due to fabrication imperfections that are difficult to avoid when dealing with the small features required to trap visible light on a nanophotonic chip.

We use a different approach which makes use of fabrication imperfections to trap light via multiple scattering and we give the first demonstration of Anderson localization of visible light on a nanophotonic chip.

By using the intrinsic photoluminescence of silicon nitride, we observe sharp resonances, a signature of the trapping of light in optical cavities, and measure quality factors approaching 10000 [4]. Such values exceed those reported for engineered $2 \mathrm{D}$ photonic crystal cavities operating at visible wavelengths.

\section{Discussion of the results}

We carry out micro-photoluminescence measurements of silicon nitride photonic crystal waveguides in which disorder is introduced by perturbing, with respect to the perfectly periodic structure, the position of the air holes next to the waveguide channel (see Fig.1). The sample is excited by a $407 \mathrm{~nm}$ continuous-wave laser and the photoluminescence signal, due to the intrinsic silicon nitride luminescence, is collected in confocal geometry and spectrally analyzed with a grating spectrometer, equipped with a silicon charge-coupled device (see Fig.1).

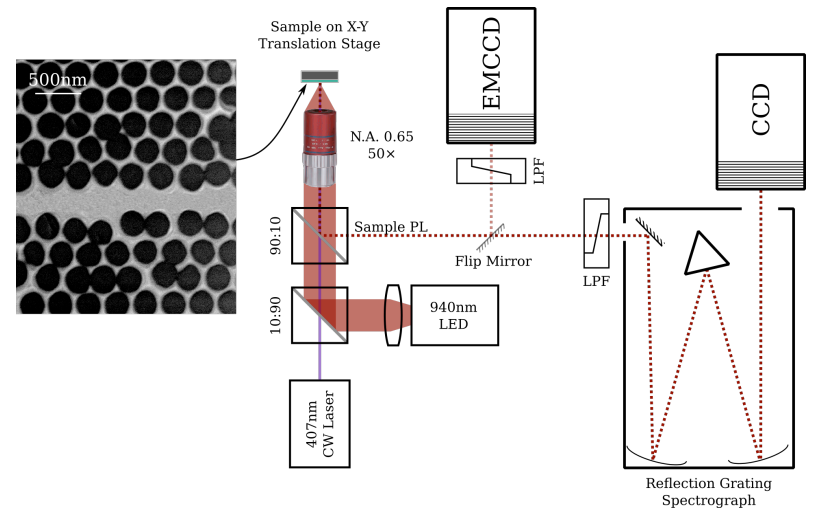

Figure 1: Scanning electron micrograph of a suspended photonic crystal waveguide where disorder is introduced by displacing the three rows of holes on either side of the waveguide. A confocal micro-photoluminescence (micro-PL) set up, with an objective with Numerical Aperture (N.A.) of 0.65 , is used to image and excite the sample, held on a xy-translation stage, with a 940-nm Light Emitting Diode (LED) or a Continuous Wave (CW) 407-nm laser, respectively. The photoluminescence is either imaged on an Electron Multiplied Charge Coupled Device (EMCCD) or sent to a spectrometer for spectral characterization, after removing the excitation light with a Long Pass Filter (LPF). 
Disorder is introduced in the system by displacing the positions of the three rows of air holes neighboring the waveguide channel by an amount obtained from a Gaussian distribution with a varying standard deviation, expressed as percentage of the photonic crystal lattice parameter.

We observe sharp spectral resonances in the photoluminescence spectra that are the signature of light confinement. When moving the excitation/collection spot along the waveguides, we observe a distribution of confined wavelengths and quality factors. We study the dependence of the quality factor of the resonances as a function of an increasing amount of disorder and observe a decrease in the measured quality factors (see Fig.2a). This trend can be explained by an increase of the out-of-plane scattering that results in greater losses when more disorder is present. For the lowest degrees of disorder, including samples where no intentional disorder is introduced, we measure quality factors exceeding 5000 and reaching a record value of 9200 (see Fig.2b), for $0 \%$ disorder, i.e. when unavoidable fabrication imperfections such as variations in the position and circularity of the air holes and in the verticality of the sidewalls are responsible for light confinement.

Our results prove that using disorder as a resource can provide quality in the light confinement exceeding that of engineered photonic crystal cavities.
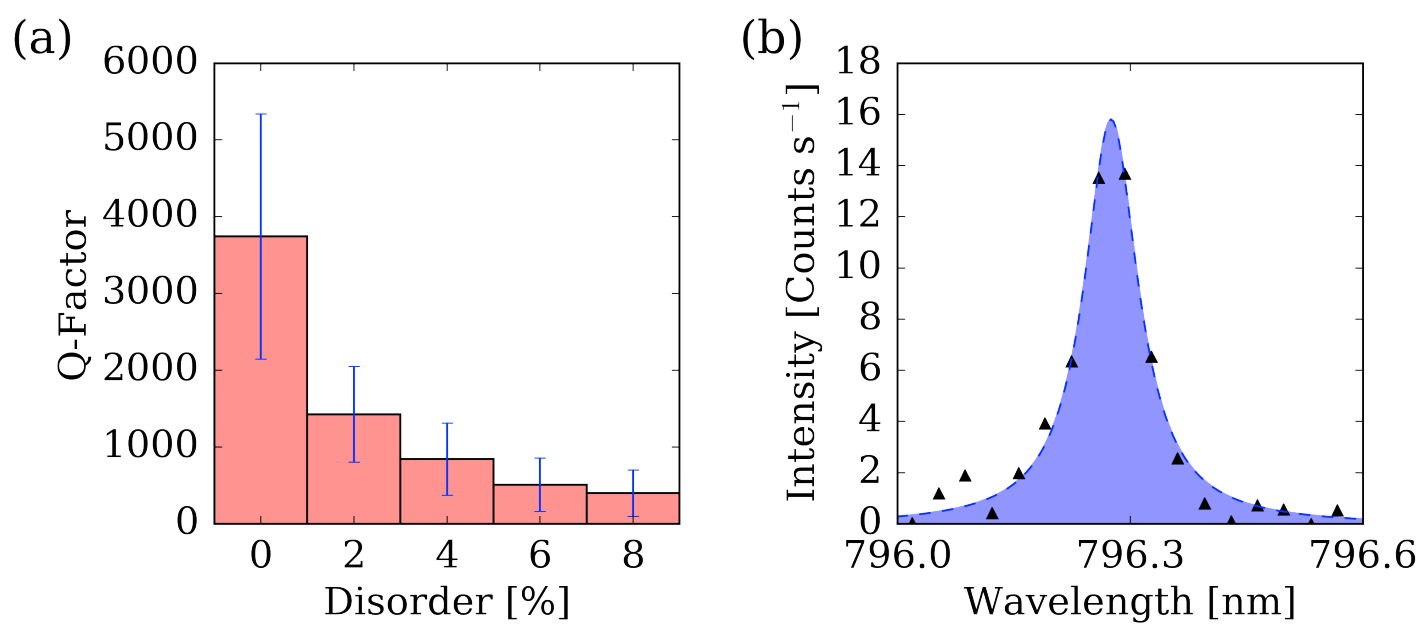

Figure 2: (a) Histograms showing the average quality factor measured on spectral resonances collected at several positions along photonic crystal waveguides with different degrees of disorder. The error bars represent one standard deviation. (b) Example of a disorder-induced optical resonance (symbols) and of its Lorentzian fit (dashed line). From the ratio between the central wavelength and the full width half maximum of the resonance, we obtain a value of the quality factor of 9200 .

\section{Conclusions}

We have fabricated disordered photonic crystal waveguides in silicon nitride and demonstrated, for the first time, Anderson localization of light at visible wavelengths on a nanophotonic chip. By means of photoluminescence spectroscopy, we observe disorder-induced optical cavities along the waveguides and measure record quality factors of the light confinement, approaching 10000.

Our results represent a new platform for enhanced light-matter interaction in the visible range of wavelengths and open the path to cavity quantum electrodynamics experiments with solid-state emitters such as defect centers in diamond, organic molecules and colloidal quantum dots.

\section{References}

[1] K.J. Vahala, Optical Microcavities, Nature 424, 839 (2003).

[2] M.M. Murshidy et al., The optical properties of hybrid organic-inorganic $L 3$ nanocavities, Journal of the Optical Society of America B 27 , 215 (2010).

[3] M. Barth et al., Modification of visible spontaneous emission with silicon nitride photonic crystal nanocavities, Optics Express 15, 17231 (2007).

[4] T. Crane, O.J. Trojak, L. Sapienza, Anderson localisation of visible light on a nanophotonic chip, https://arxiv.org/abs/1605.08614v2 (2016). 